\title{
5G Network Model Analysis by NS2 Simulation: An Extensive Review
}

\author{
${ }^{1} \mathrm{~N}$. Thirupathi Rao, ${ }^{1}$ Debnath Bhattacharyya and ${ }^{2}$ Tai-Hoon Kim \\ ${ }^{1}$ Department of Computer Science and Engineering, \\ Vignan's Institute of Information Technology (A), Visakhapatnam, Andhra Pradesh (AP), India \\ nakkathiru@gmail.com, 9966968368, debnathb@gmail.com, 9492040744 \\ ${ }^{2}$ Sungshin Women's University, Bomun-ro 34da-gil, Seongbuk-gu, Seoul, Korea \\ taihoonn@daum.net
}

\begin{abstract}
G wireless networks are the latest communication technologies which were in the move of changing the entire current existing technologies and bringing new era of communication speeds for data and class transfers. While there has been sure agreement on the general prerequisites of $5 \mathrm{G}$ remote frameworks, different empowering remote innovations have been considered and concentrated on accomplishing these execution targets. This study displays a system show for cutting edge cell systems intended to meet the dangerous needs of versatile information while limiting vitality utilization. The joined utilization of remote edge storing and coded multicasting has been appeared to be a promising way to deal with multicast transmissions, data transfer capacity proficiency picks up the worldview depends on two standards: reserving and multicasting. Keeping in mind the end goal to improve utilization of the accessible store space, the current system is upgraded and multicast transmission is considered. Some detailed review about the performance of the considered $5 \mathrm{G}$ wireless communication network model had been discussed with the help of some simulation results.
\end{abstract}

Key words: Communication networks, $5 \mathrm{G}$ networks, caching, multicast, routing, model

\section{INTRODUCTION}

The development of better versatile broadband facility and utilization is increasing the business to look at how systems can be utilised to meet future advanced limit and execution requests. The $5 \mathrm{G}$ should be the arrangement of functional parts expected to deal with these necessities (Pizzi et al., 2018a, b). The 5G underscores the advancement of existing strategies to enhance limit, joined with development in radio innovation even an adjustment in framework plan standards. Future remote access will stretch out past individuals, supporting availability for any component that may profit by being associated. One of the key inspirations for $5 \mathrm{G}$ is to give universal, fast, astounding remote broadband scope to meet societal and mechanical needs by past 2020 (Pizzi et al., 2018a, b). The positive test is to empower a large group of various stages ready to cooperate as a consistent to a great extent programming controlled and sufficiently adaptable to help any use design. More bound together and institutionalized settled access arrangements would permit substantially higher volumes and in this way higher mix densities, much lower cost and reduced vitality utilization (Guo et al., 2018).
We are observing an uncommon overall development of versatile information movement that relied upon to proceed at a yearly rate of throughout the following years by achieving exabytes every month. To deal with this information wave, the rising fifth era $5 \mathrm{G}$ frameworks need to enhance the system execution as far as vitality utilization, throughput and client experienced deferral and in the meantime improve an utilization of the system assets (Montalban et al., 2018). With the introduction of sensible hand-held gadgets, client demands for versatile broadband are encountering accomplice degree uncommon climb. The strong advancement of information exchange applications like video spouting and transmission report sharing are as of now pushing the breaking points of current cell structures. Inside the next decade, media-rich versatile applications like telecom and 3D optics would require information rates just horrendous with fourth period (4G) systems (Poularakis et al., 2014). The relentlessly creating enthusiasm for higher information rates and limit require odd theory for taking after period $(5 \mathrm{G})$ cell systems.

\section{Literature review}

Multicast-mindful caching for small-cell networks: The sending of little cells is relied upon to increase

Corresponding Author: N. Thirupathi Rao, Department of Computer Science and Engineering, Vignan's Institute of Information Technology (A), Visakhapatnam, Andhra Pradesh (AP), India nakkathiru@gmail.com, 9966968368 
tremendous force sooner than later as an answer for dealing with the soaring versatile information request development. Neighborhood reserving of mainstream records at the little cell base stations has been late proposed, going for decreasing the movement brought about while exchanging the content from the centre system to the clients (Anonymous, 2014, 2015a, b). In this study, a novel reserving approach that can accomplish altogether bring down activity contrasted with the customary storing plans was examined. The current outline approach precisely considers the way that an administrator can serve the solicitations for a similar record that occur at adjacent circumstances using a single multicast transmission. The last brings about less activity as the asked for the record is transmitted to the clients just once as opposed to with many unicast transmissions (Andrews, 2013). Methodical examinations show the adequacy of our approach when contrasted with the current storing plans.

Mobility report: on the pulse of networked society: Spiking Neural Networks (SNNs) have turned into a vital research subject because of new revelations and advances in neurophysiology which expresses the data among neurons is traded using heartbeats or spikes. FPGAs generally utilized for actualizing superior computerized equipment frameworks because of its adaptability and they are appropriate for the usage of frameworks with a high level of parallelism (Xu et al., 2015). FPGAs have turned into a vital apparatus, since, first grain computerized components valuable for productive equipment execution of SNNs are given, making FPGA gadget appropriate for actualizing SNNs. SNNs are less equipment covetous and the idea of the beat preparing is appropriate to the computerized handling pieces of the FPGA gadgets. A few PC vision applications have been actualized utilising SNNs (Erman et al., 2011). A standout amongst the most basic undertakings in $\mathrm{PC}$ vision is picture grouping. In this study, equipment engineering for executing picture bunching utilising SNNs is accounted for and observed.

\section{MATERIALS AND METHODS}

NS2: NS are a discrete occasion test system focused on systems administration. NS give generous help to a reenactment of $\mathrm{TCP}$, directing and multicast conventions over wired and remote systems. NS started as a variation of the REAL test system in 1989 and have developed generously finished a previous couple of years ( $\mathrm{Xu}$ et al., 2015). In 1995, NS advancement was bolstered by DARPA through the VINT venture at LBL, Xerox PARC, UCB and USC/ISI. At present, NS improvement is bolstered through DARPA with SAMAN and NSF with CONSER, both as a team with different specialists including ACIRI (Erman et al., 2011). NS have included continuously substantial commitments from different analysts, including remote code from the UCB Needless and CMU Monarch undertakings and Sun Microsystems (Erman et al., 2011). Then, again an extensive piece of system examine includes somewhat shifting parameters or designs or rapidly investigating various situations. In these cases, cycle time is more vital. Since, arrangement runs once, the run-time of this piece of the errand is less imperative (Ramanan et al., 2013).

Existing system: 4G technology is an integrated, global network that will be able to provide a comprehensive IP solution where voice, data and streamed multimedia can be given to users on an "Anytime, Anywhere" basis. It is intended to support broadband performance and enable voice/video multimedia applications. At present, LTE (Long Term Evolution) and WiMAX (Worldwide interoperability for Microwave Access) are the two technologies considered as candidates to achieve the $4 \mathrm{G}$ wireless performance objectives. The $4 \mathrm{G}$ will be a fully IP-based integrated system. $4 \mathrm{G}$ will be capable of providing between $100 \mathrm{Mbit} / \mathrm{sec}$ and $1 \mathrm{Gbit} / \mathrm{sec}$ speeds both indoors and outdoors with premium quality and high security.

Proposed system: Heterogeneous cell systems show that existing systems are storing and multicast for the administration of the portable clients. Stores can introduce at little cell Base Stations (SBSs) like picocells and femtocells, focusing to offload movement from the assembled full-scale cell base station (MBS). Estimation examines uncovered up to $66 \%$ reduction in arrange activity by utilising reserving in $3 \mathrm{G}$ and $4 \mathrm{G}$ systems. In the interim, the remote business started to popularize frameworks that help to reserve. Numerous administrators exploit multicast to effectively use the available data transfer capacity of their systems in conveying a similar substance to different collectors. For instance, multicast regularly utilized for conveying supported substance, e.g., versatile notices in specific areas, downloading news, securities exchange reports, climate and games refreshes. In the interim, multicast has fused in 3GPP details in which the proposed innovation for LTE is called Evolved Multimedia Broadcast and Multicast Services (eMBMS). This innovation used for different cells where the transmission crosswise over them is synchronous utilising a common bearer recurrence. Henceforth, a simulation study had conducted such that to analyze the performance of such systems with various protocols and tried to analyze the performance of the model. 
System design: The Heterogeneous Cellular Network ( $\mathrm{HCN}$ ) engineering will be composed where it comprises a Macro cell Base Station (MBS) and various Small cell Base Stations (SBS) and additionally transfers. Stores can be introduced at SBS and focusing to offload activity from the gathered MBS. Store loaded with mainstream content records at Evolved Packet Core (EPC) or the Radio Access Network (RAN). SBS permit spatial reuse thus they can increment fundamentally limit and scope. Reserving content at the remote edge has increased extensive footing as a promising procedure for future remote systems. Edge storing originates from the likelihood of fundamentally diminishing the backhaul utilization by conveying the substance nearer to the end clients. Various researches have examined the potential advantages of reserving content in thickly conveyed SBS furnished with capacity abilities. A few approaches to evaluate the execution of reserving have proposed, e.g., from a data theoretic viewpoint and a blackout likelihood perspective. One measure of extraordinary intrigue is the vitality Effectiveness (EE) which has increased great consideration over the most recent couple of years. A simulation mechanism was chosen such that to analyze the performance $f$ the considered model with various protocols with various set of nodes at various situations and scenarios (3GPP., 2019).

\section{Ad hoc On-demand Distance Vector (AODV) routing} protocol: The ad hoc on-request distance vector directing convention acquires the great highlights of both DSDV and DSR. The AODV steering convention utilises a responsive way to deal with discovering courses and a proactive approach for distinguishing the latest way. All the more particularly, it discovers courses utilising the course revelation process like DSR and utilizations goal succession numbers to register new courses. The two stages examined in more detail.

Route discovery: Amid the course disclosure process, the source hub communicates RREQ bundles like DSR. The RREQ parcel contains the Source Identifier (SID), the goal-identifier (DD), the source grouping number (SSeq), the goal arrangement number (DEQ), the communicate identifier (BID) and TTL fields. At the point when a transitional hub gets an RREQ bundle, it either advances it or readies a Route Reply (RREP) parcel if it has a substantial course to the goal in its reserve. The (SID, BID) combine is utilised to decide whether a specific RREQ has just been gotten keeping in mind the end goal to dispense with copies. Each middle of the road hub enters the past hub's address and its BID while sending an RREQ parcel. The hub likewise keeps up a clock related to each section to erase an RREQ bundle, if the answer is not gotten before it terminates. At whatever point a hub gets an RREP bundle, it stores the data of the past hub keeping in mind the end goal to forward the bundle to it as the next jump towards the goal.

\section{RESULTS AND DISCUSSION}

Figure 1, the stating of the simulation process and the selection of various number of node sin the network can be observed in detail.

Figure 2, the initialization process of various nodes can be observed. In order to identify the performance of the network, the initialization of some set of nodes is required.

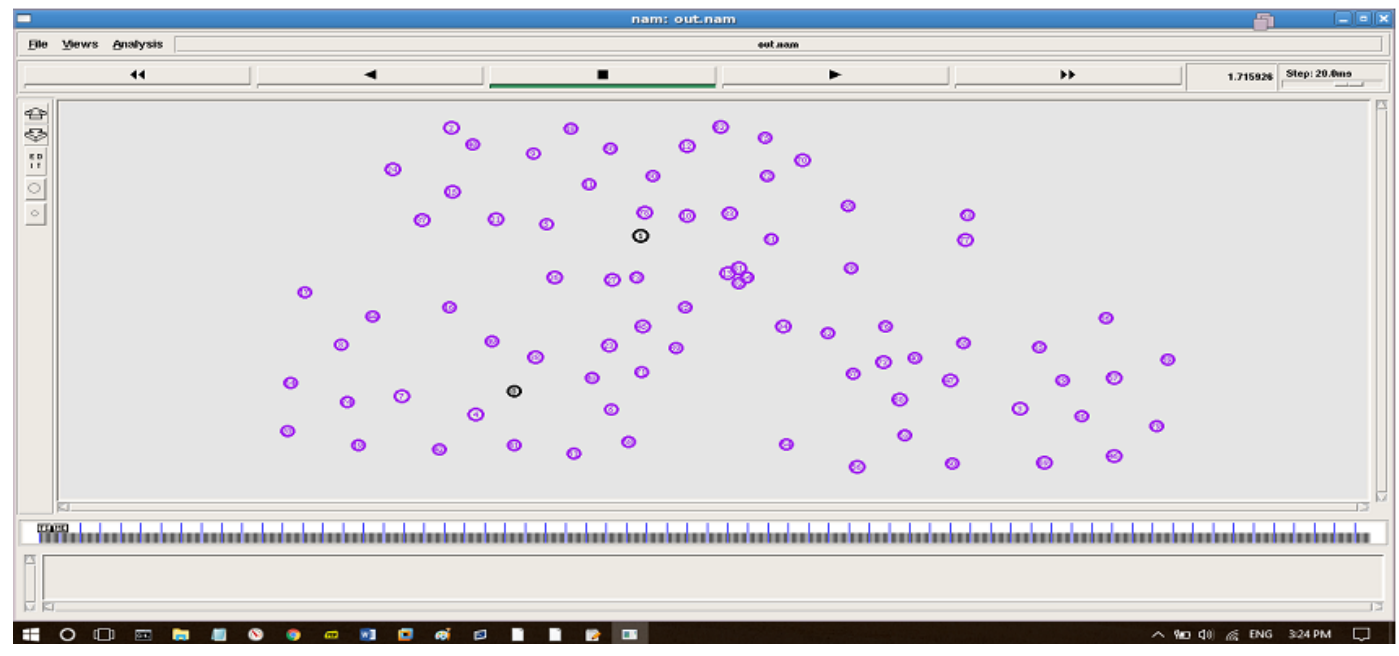

Fig. 1: Starting simulation 


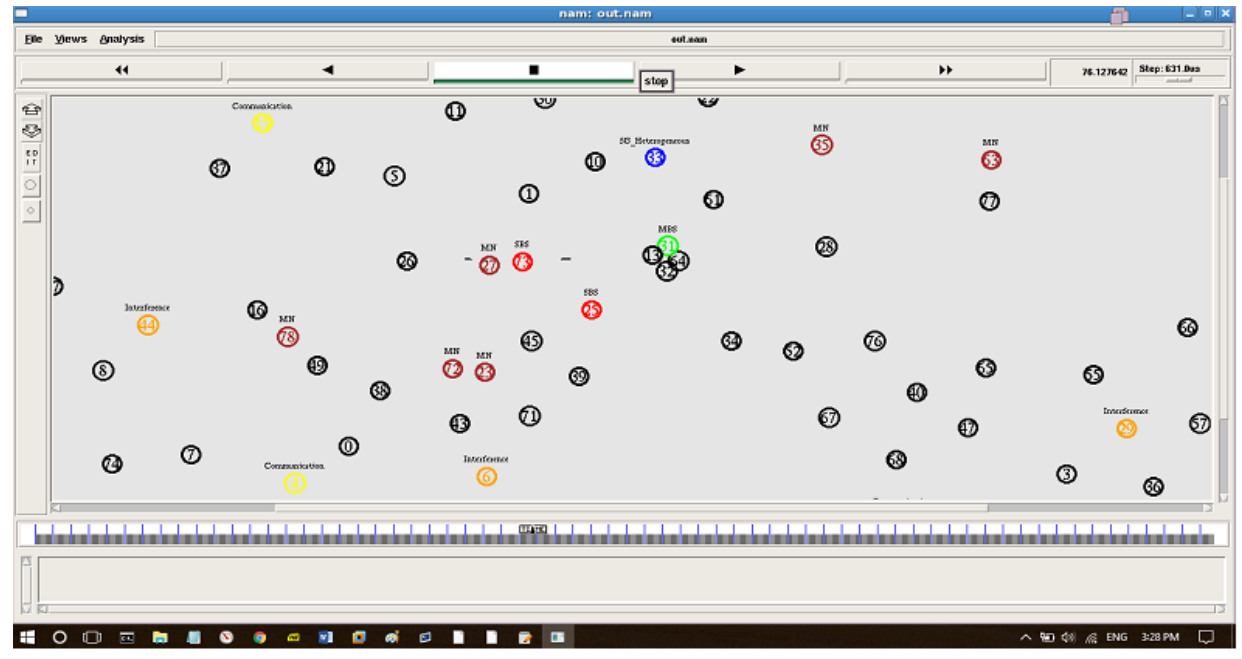

Fig. 2: Initialization of mobile nodes

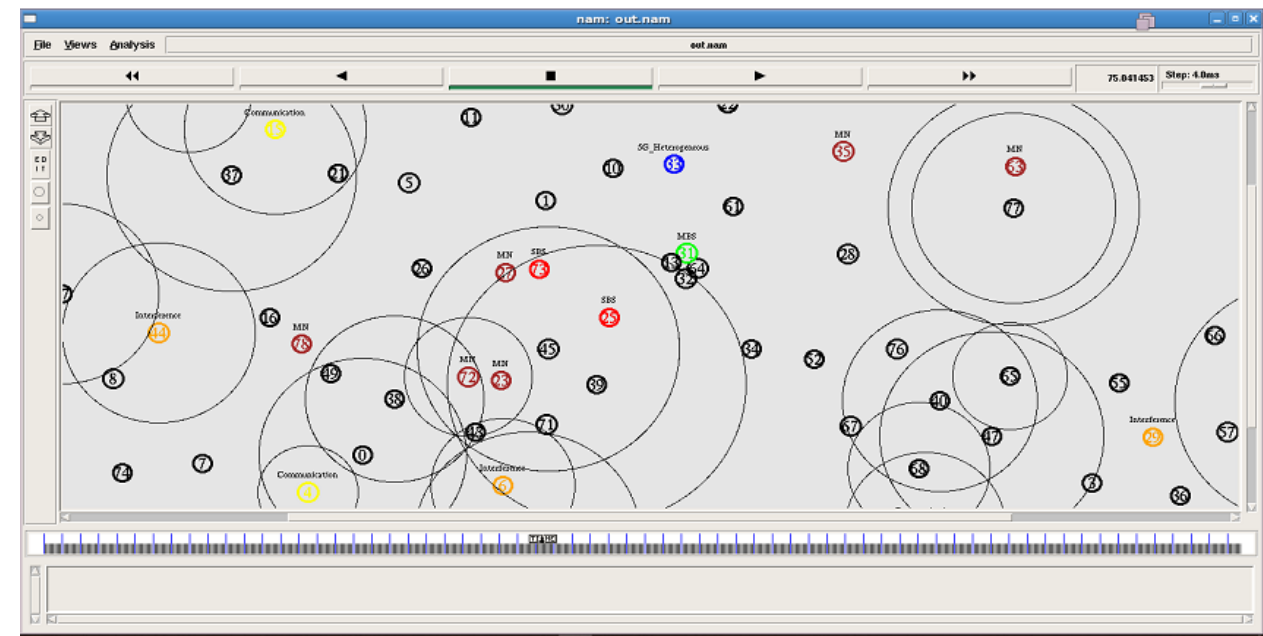

\#○吅

Fig. 3: Routing tables are updating in all nodes of the network

Figure 3 , the routing tables are updated with the set of nodes and the number of routes available and also the set of nodes available for each route. All these routing tables are updated from time to time.

Figure 4, the serving of one node by the other node can be observed. Here, in this particular case, node 72 is serving to the small base station 34 and can be clearly observed in the above image.

Figure 5, the comparison of average transmission rates in the considered networks with various routing protocols was displayed. The performance of these protocols on the currently considered model can be observed in detail in the form of graphical representations.

Figure 6 , the cache success rate and the cache probability rates in small base stations also can be observed in detail. Various nodes and their success rate in terms of cache process can be observed in detail.

Figure 7 , the average transmission rates of the currently considered models are observed and their performance can be observed in the form of graphical representation.

At first, the selection and creation of nodes in the network was done in the simulator. The initialization of nodes will be started in the next phase of the execution process. Routing tables with the updated values will be updated and the same thing can be observed in the above image No. 3. By applying the AODV routing protocol to study the performance of the considered network model with various parameters were discussed in detail and 


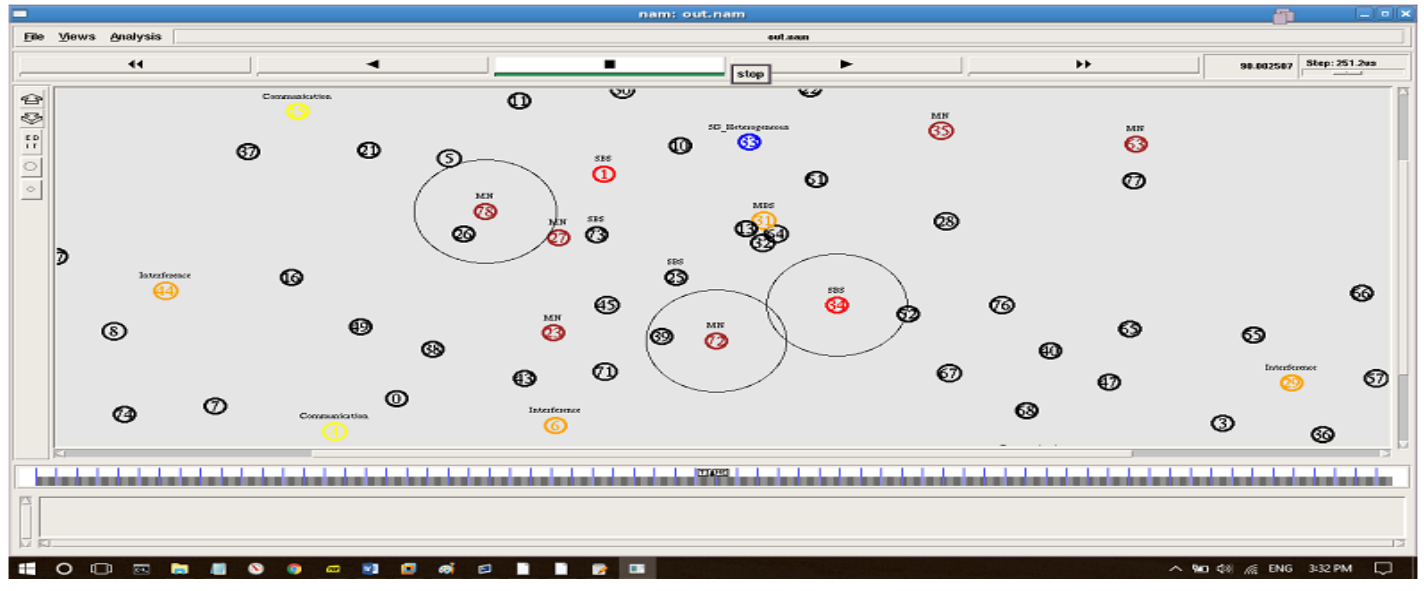

Fig. 4: Mobile node 72 is serving by small base station 34

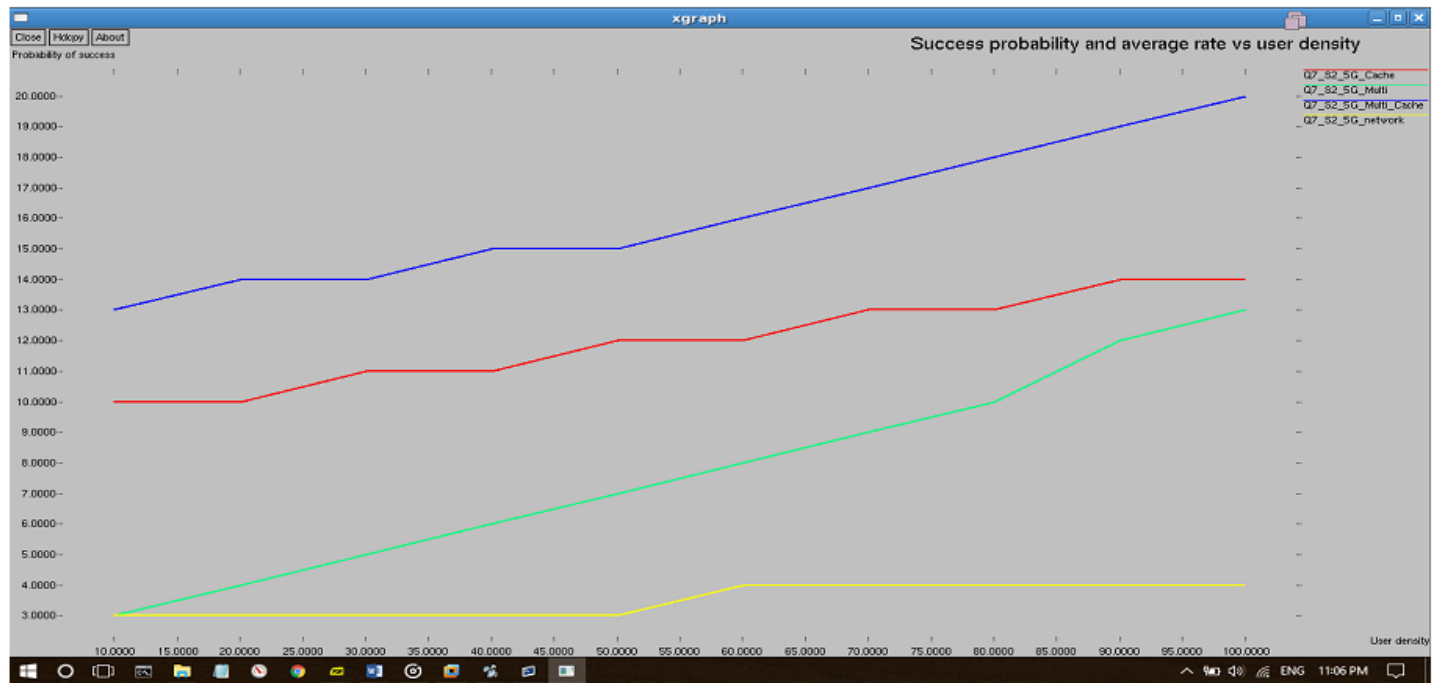

Fig. 5: Comparison of average transmission rate in $5 \mathrm{G}$ techniques

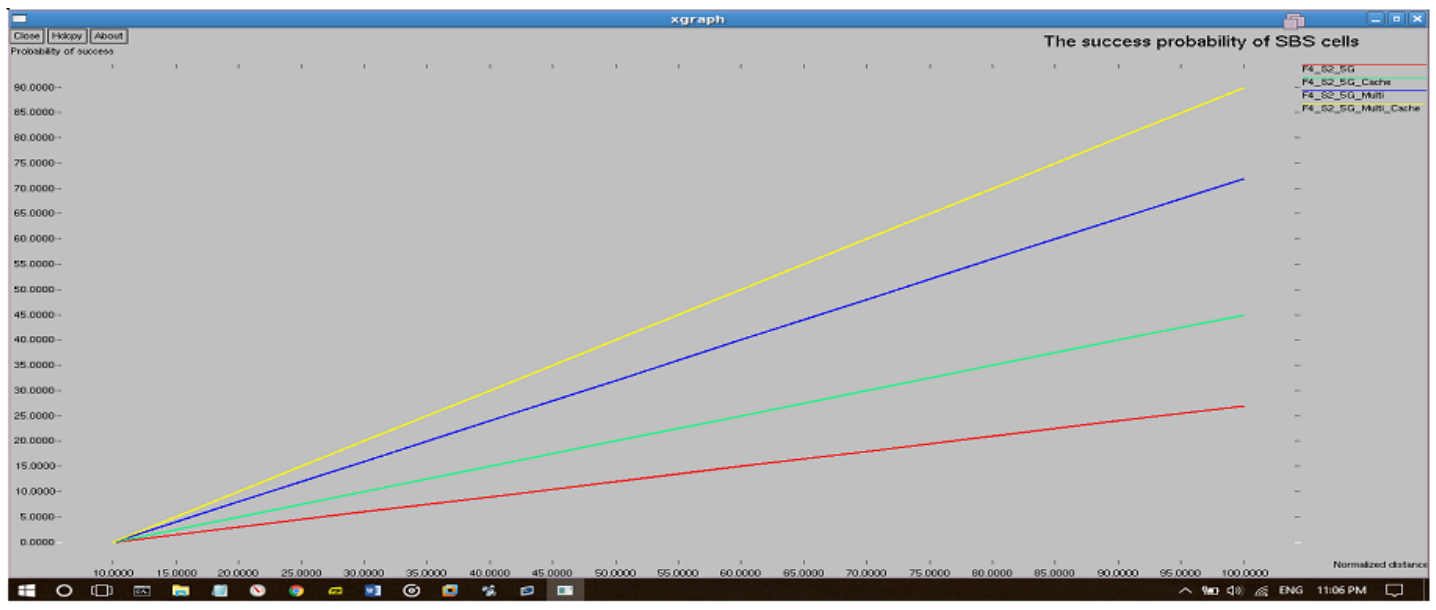

Fig. 6: Cache success probability in SBS's 


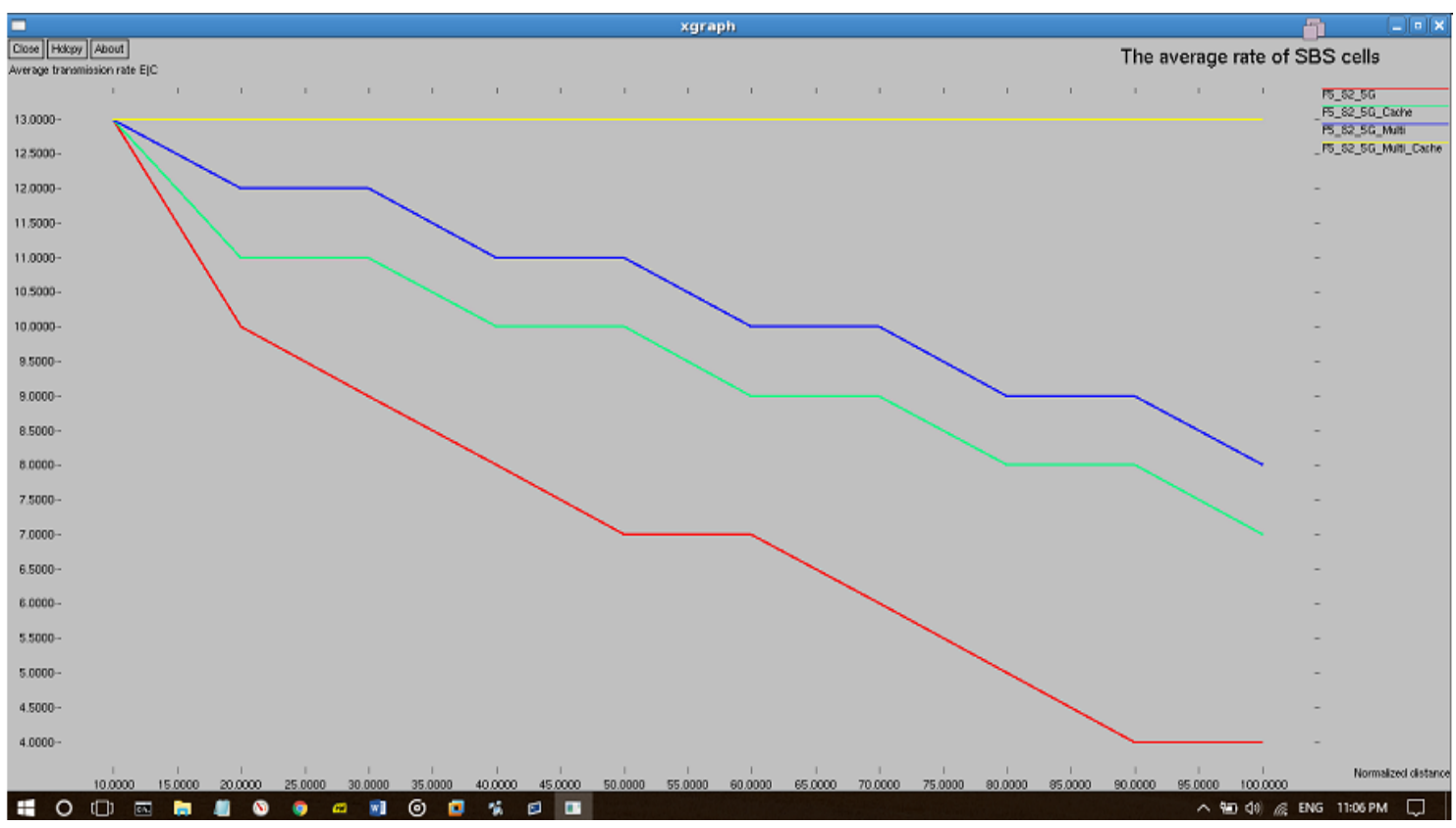

Fig. 7: The average transmission rate in SBS's

displayed in the form of the graphical representation. Various performance metrics like the average transmission rate and the average transmission rate in small base stations was also discussed in detail. The graphical representation can be observed in the above 3 images.

\section{CONCLUSION}

In the present study, the researchers had proposed a $5 \mathrm{G}$ network simulation model that can reduce the vitality expenses of broad-scale versatile information needs in $5 \mathrm{G}$ remote systems. In opposition to conventional reserving plans, common storing procedures essentially convey common substance to close clients and the currently considered reserving technique is all around intended to make additional utilization of multicast of data. The outcomes from the considered model demonstrate that portfolio is reserving and multicast can decrease vitality costs when the interest for deferred resistance content is enormous. At the point when the client endures $3 \mathrm{~min}$ of postponement with the steepness of the substance gets to show and further increment, the current store program income of $19 \%$. When all said in done, the current research can be viewed as an endeavor to methodically join reserving and multicast as methods for enhancing vitality productivity in 5G remote systems.

\section{ACKNOWLEDGEMENTS}

We would like to thanks to SoCoRI Society, Korea, who gave us the research support and opportunity to conduct this research which also helped us in doing a lot of research activities and we came to know about so many new things we are really thankful to them.

\section{REFERENCES}

3GPP., 2019. The mobile broadband standard. 3GPP Sophia Antipolis, France.

Andrews, J.G., 2013. Seven ways that HetNets are a cellular paradigm shift. IEEE. Commun. Mag., 51: 136144.

Anonymous, 2014. NSN adds ChinaCache smarts to liquid applications. Light Reading, New York, USA. https://www.lightreading.com/mobile/4g-lte/-nsnadds-chinacache-smarts-to-liquid-applications-/d/did $/ 708280$

Anonymous, 2015a. Altobridge debuts Intel-based network edge small cells caching solution. Mobile Europe, Europe. https://www.mobileeurope. co.uk/Press-Wire/altobridge-debuts-intel-basedhierarchical-network-edge-caching-solution

Anonymous, 2015b. Ericsson mobility report: On the pulse of the networked society. GSM Association, London, UK. 
Erman, J., A. Gerber, M. Hajiaghayi, D. Pei and S. Sen et al., 2011. To cache or not to cache: The $3 \mathrm{G}$ case. IEEE. Internet Comput., 15: 27-34.

Guo, W., M. Fuentes, L. Christodoulou and B. Mouhouche, 2018. Roads to multimedia broadcast multicast services in $5 \mathrm{G}$ new radio. Proceedings of the 2018 IEEE International Symposium on Broadband Multimedia Systems and Broadcasting (BMSB), June 6-8, 2018, IEEE, Valencia, Spain, ISBN:978-1-5386-4730-1, pp: 1-5.

Montalban, J., P. Scopelliti, M. Fadda, E. Iradier and C. Desogus et al., 2018. Multimedia multicast services in $5 \mathrm{G}$ networks: Subgrouping and nonorthogonal multiple access techniques. IEEE. Commun. Mag., 56: 91-95.

Ofweek, 2014. China Telecom successfully deployed LTE eMBMS. OFweek Industrial B2B Marketplace, Shenzhen, China.

Pizzi, S., C. Suraci, L. Militano, A. Orsino and A. Molinaro et al., 2018b. Enabling trustworthy multicast wireless services through D2D communications in 5G networks. Future Inter., 10: $2-15$.
Pizzi, S., F. Rinaldi, A. Molinaro, A. Iera and G. Araniti, 2018a. Energy-efficient multicast service delivery exploiting single frequency device-to-device communications in $5 \mathrm{G}$ new radio systems. Sens., 18: 1-15.

Poularakis, K., G. Iosifidis, V. Sourlas and L. Tassiulas, 2014. Multicast-aware caching for small cell networks. Proceedings of the 2014 IEEE International Conference on Wireless Communications and Networking Conference (WCNC), April 6-9, 2014, IEEE, Istanbul, Turkey, ISBN:978-1-4799-3083-8, pp: 2300-2305.

Ramanan, B.A., L.M. Drabeck, M. Haner, N. Nithi and T.E. Klein et al., 2013. Cacheability analysis of HTTP traffic in an operational LTE network. Proceedings of the International Symposium on Wireless Telecommunications (WTS), April 17-19, 2013, IEEE, Phoenix, Arizona, USA., ISBN:978-1-4673-5011-2, pp: 1-8.

Xu, Y., J. Wang, Q. Wu, Z. Du and L. Shen et al., 2015. A game-theoretic perspective on self-organizing optimization for cognitive small cells. IEEE. Commun. Mag., 53: 100-108. 